\title{
The $q$-Difference Theorems for Meromorphic Functions of Several Variables
}

\author{
Zhi-Tao Wen \\ Department of Mathematics, Taiyuan University of Technology, Yingze West Street, No. 79, Taiyuan 030024, China \\ Correspondence should be addressed to Zhi-Tao Wen; zhitaowen@gmail.com
}

Received 28 March 2014; Revised 9 May 2014; Accepted 22 May 2014; Published 1 June 2014

Academic Editor: Bashir Ahmad

Copyright (C) 2014 Zhi-Tao Wen. This is an open access article distributed under the Creative Commons Attribution License, which permits unrestricted use, distribution, and reproduction in any medium, provided the original work is properly cited.

We investigate $q$-shift analogue of the lemma on logarithmic derivative of several variables. Let $f$ be a meromorphic function in $\mathbb{C}^{n}$ of zero order such that $f(0) \neq 0, \infty$, and let $q \in \mathbb{C}^{n} \backslash\{0\}$. Then we have $m(r, f(q z) / f(z))=o(T(r, f))$ on a set of logarithmic density 1. The $q$-shift analogue of the first and the second main theorems of Nevanlinna theory of several variables and their applications is also shown.

\section{Introduction}

The lemma on logarithmic derivative is a basic tool of Nevanlinna theory; see [1]. It is widely used in value distribution of meromorphic functions [2], differential equations in the complex plane [3], and so forth. The first generalization of the lemma on the logarithmic derivative to several complex variables is given by Vitter [4]. Another proof is given by Biancofiore and Stoll in [5]. Ye obtains a sharp bound for the lemma on logarithmic derivative of several complex variables in [6]. In [7], Li improves the lemma on logarithmic derivative of several variables without exceptional intervals, which makes a significant difference from other estimates.

Essentially prompted by the recent interest in discrete Painlevé equations, difference analogues of the lemma on logarithmic derivative appeared to be useful in a number of applications, in particular in complex difference equations; see [8-10]. Later, Korhonen studies the difference analogues of the lemma on logarithmic derivative of several variables in [11]. For $q$-difference version, Barnett et al. obtain $q$ difference analogue of the lemma on logarithmic derivative in the complex plane in [12].

In this paper, our aim is to generalize the result in [12] to several variables. For any two constants $z$ and $z^{\prime}$ in $\mathbb{C}^{n}$, if $z=\left(z_{1}, \ldots, z_{n}\right)$ and $z^{\prime}=\left(z_{1}^{\prime}, \ldots, z_{n}^{\prime}\right)$, then we denote $z_{1} z_{1}^{\prime}=$ $\left(z_{1} z_{1}^{\prime}, \ldots, z_{n} z_{n}^{\prime}\right)$. Let us state one of the main results as follows, which is $q$-difference analogue of the lemma on logarithmic derivative of several variables.
Theorem 1. Let $f$ be a meromorphic function in $\mathbb{C}^{n}$ of zero order such that $f(0) \neq 0, \infty$, and let $q \in \mathbb{C}^{n} \backslash\{0\}$. Then

$$
m\left(r, \frac{f(q z)}{f(z)}\right)=o(T(r, f))
$$

on a set of logarithmic density 1.

Concerning the sharpness of Theorem 1 , there is an example in [12, page 459] to show that zero order cannot be replaced by any positive order in the statement of Theorem 1 .

The remainder of the paper is organized as follows. In Section 2, we prove the theorem of $q$-difference analogue of the lemma on logarithmic derivative of several variables. In Section 3, we present the $q$-shift analogue of the first and the second main theorems of Nevanlinna theory of several variables. In Section 4, we give three important theorems: uniqueness theorem of meromorphic function with its $q$-shift of several variables, $q$-shift analogues of the Clunie lemma, and Mohon'ho lemma of several variables.

\section{2. q-Difference Logarithmic Derivative Analogue}

The purpose of this section is to present $q$-difference analogues of the lemma on logarithmic derivative in several complex variables. Let us recall some of the standard notations of Nevanlinna theory in $\mathbb{C}^{n}$. 
Let $M$ be a connected complex manifold of dimension $n$ and let

$$
A(M)=\sum_{n=0}^{2 m} A^{n}(M)
$$

be the graded ring of complex valued differential forms on $M$. Each set $A^{n}(M)$ can be split into a direct sum

$$
A^{n}(M)=\sum_{p+q=n} A^{p, q}(M)
$$

where $A^{p, q}(M)$ is the forms of type $(p, q)$. The differential operators $d$ and $d^{c}$ on $A(M)$ are defined as

$$
d:=\partial+\bar{\partial}, \quad d^{c}:=\frac{1}{4 \pi i}(\partial-\bar{\partial}),
$$

where

$$
\begin{aligned}
& \partial: A^{p, q}(M) \longrightarrow A^{p+1, q}(M), \\
& \bar{\partial}: A^{p, q}(M) \longrightarrow A^{p, q+1}(M) .
\end{aligned}
$$

Let $z=\left(z_{1}, \ldots, z_{n}\right) \in \mathbb{C}^{n}$, and let $r>0$ and fix $a=$ $\left(a_{1}, \ldots, a_{n}\right) \in \mathbb{C}^{n}$; an exhaustion function $\tau_{a, \mathbb{C}^{n}}$ of $\mathbb{C}^{n}$ is defined by

$$
\tau_{a, \mathbb{C}^{n}}(z)=|z-a|^{2}=\sum_{j=1}^{m}\left|z_{j}-a_{j}\right|^{2} .
$$

Usually, we let $a=0$ be the origin and set $\tau_{m}(z)=\tau_{0, \mathbb{C}^{n}}(z)$. Let us define a positive measure $\sigma_{n}(z)$ with total measure one on the boundary $\partial B_{n}(r):=\left\{z \in \mathbb{C}^{n}:|z|=r\right\}$ of the ball $B_{n}(r):=\left\{z \in \mathbb{C}^{n}:|z|<r\right\}$ as

$$
\omega_{n}(z):=d d^{c} \log \tau_{n}, \quad \sigma_{n}(z):=d^{c} \log \tau_{n} \wedge \omega_{n}^{n-1}(z) .
$$

In addition, we define

$$
v_{n}(z):=d d^{c} \tau_{n}(z), \quad \rho_{n}(z): v_{n}^{n}(z) .
$$

It follows that $\rho_{n}(z)$ is the Lebesgue measure on $\mathbb{C}^{n}$ normalized such that the ball $B_{n}(r)$ has measure $r^{2 n}$.

Let us start with the one-dimensional case at first, the following result is based on the proof of [12, Lemma 5.1].

Lemma 2. Let $f$ be a meromorphic function in $\mathbb{C}$ such that $f(0) \neq 0, \infty$, and let $q \in \mathbb{C} \backslash\{0\}$. Then

$$
\begin{aligned}
& \int_{\partial B_{1}(r)} \log ^{+}\left|\frac{f(q z)}{f(z)}\right| \sigma_{1}(z) \\
& \leq\left(\frac{|q-1|^{\delta}\left(|q|^{\delta}+1\right)}{\delta(1-\delta)|q|^{\delta}}+\frac{|q-1| r}{\lambda-|q| r}+\frac{|q-1| r}{\lambda-r}\right) \\
& \times\left(n(\lambda, f)+n\left(\lambda, \frac{1}{f}\right)\right) \\
&+\frac{2|q-1| r \lambda}{(\lambda-r)(\lambda-|q| r)}\left(m(\lambda, f)+m\left(\lambda, \frac{1}{f}\right)\right),
\end{aligned}
$$

where $z=r e^{i \phi}, \lambda>\max \{r,|q| r\}$, and $0<\delta<1$.
Let $\tau_{m}=\tau_{0, \mathbb{C}^{n}}$ be the parabolic exhaustion of $\mathbb{C}^{n}$ and define a function on $\bar{B}_{n-1}(r)$ by

$$
p_{r}(\omega)=\sqrt{r^{2}-\tau_{n-1}(\omega)}=\sqrt{r^{2}-|\omega|^{2}}
$$

For the following discussion, we will need two lemmas from Stoll.

Lemma 3 (see [13, Lemma 1.29]). Let $r>0$, and let $h$ be a function on $\partial B_{n}(r)$ such that $\sigma_{n}$ is integrable over $\partial B_{n}(r)$. Then

$$
\begin{aligned}
\int_{\partial B_{n}(r)} & h(z) \sigma_{n}(z) \\
= & \frac{1}{r^{2 n-2}} \int_{\bar{B}_{n-1}(r)} \int_{\partial B_{1}\left(p_{r}(w)\right)} h(\omega, \zeta) \sigma_{1}(\zeta) \rho_{n-1}(\omega) .
\end{aligned}
$$

Let $f$ be a nonconstant meromorphic function on $\mathbb{C}^{n}$, take $\omega \in \mathbb{C}^{n-1}$, and define the holomorphic map $j_{m}: \mathbb{C} \rightarrow$ $\mathbb{C}^{n}$ by $j_{w}(z)=(w, z)$; thus $f_{\omega}(z)=f(\omega, z)$.

Lemma 4 (see [13, Lemma 1.30]). Let $f$ be a nonconstant meromorphic function in $\mathbb{C}^{n}$ and take $a \in \mathbb{P}^{1}$. If $r>0$, then

$$
\begin{aligned}
& \frac{1}{r^{2 n-2}} \int_{\partial B_{n}(r)} n\left(p_{r}(\omega), \frac{1}{f_{\omega}-a}\right) \sigma_{n-1}(\omega) \leq n\left(r, \frac{1}{f-a}\right), \\
& \frac{1}{r^{2 n-2}} \int_{\partial B_{n}(r)} m\left(p_{r}(\omega), \frac{1}{f_{\omega}-a}\right) \sigma_{n-1}(\omega)=m\left(r, \frac{1}{f-a}\right) .
\end{aligned}
$$

We proceed to generalize Lemma 2 to several complex variables by using Lemmas 3 and 4 .

Lemma 5. Let $f$ be a meromorphic function in $\mathbb{C}^{n}$ such that $f(0) \neq 0, \infty$, and let $\widetilde{q_{j}}:=\left(1, \ldots, q_{j}, \ldots, 1\right)$. Then

$$
\begin{aligned}
& \int_{\partial B_{n}(r)} \log ^{+}\left|\frac{f\left(\widetilde{q_{j}} z\right)}{f(z)}\right| \sigma_{n}(z) \\
& \leq\left(\frac{\left|\widetilde{q_{j}}-1\right|^{\delta}\left(\left|\widetilde{q_{j}}\right|^{\delta}+1\right)}{\delta(1-\delta)\left|\widetilde{q_{j}}\right|^{\delta}}+\frac{\left|\widetilde{q_{j}}-1\right| r}{R-\left|\widetilde{q_{j}}\right| r}+\frac{\left|\widetilde{q_{j}}-1\right| r}{R-r}\right) \\
& \quad \times\left(n(R, f)+n\left(R, \frac{1}{f}\right)\right) \\
& \quad+\frac{2|q-1| r R}{(R-r)(R-|q| r)}\left(m(R, f)+m\left(R, \frac{1}{f}\right)\right),
\end{aligned}
$$

where $R>\max \left\{r,\left|\widetilde{q_{j}}\right| r\right\}$ and $0<\delta<1$. 
Proof. By applying Lemma 3 with $h(z)=\log ^{+}\left|f\left(\widetilde{q_{j}} z\right) / f(z)\right|$, we obtain

$$
\begin{aligned}
& \int_{\partial B_{n}(r)} \log ^{+}\left|\frac{f\left(\widetilde{q_{j}} z\right)}{f(z)}\right| \sigma_{n}(z) \\
& =\frac{1}{r^{2 n-2}} \int_{\bar{B}_{n-1}(r)} \int_{\partial B_{1}\left(p_{r}(\omega)\right)} \log ^{+}\left|\frac{f_{\omega}\left(\widetilde{q_{j}} z\right)}{f_{\omega}(z)}\right| \sigma_{1}(\zeta) \rho_{n-1}(\omega) \\
& \leq \frac{1}{r^{2 n-2}} \int_{\bar{B}_{n-1}(r)}\left(\frac{\left|\widetilde{q_{j}}-1\right|^{\delta}\left(\left|\widetilde{q_{j}}\right|^{\delta}+1\right)}{\delta(1-\delta)\left|\widetilde{q_{j}}\right|^{\delta}}\right. \\
& \quad+\frac{\left|\widetilde{q_{j}}-1\right| p_{r}(\omega)}{p_{R}(\omega)-\left|\widetilde{q_{j}}\right| p_{r}(\omega)} \\
& \left.\quad+\frac{\left|\widetilde{q_{j}}-1\right| p_{r}(\omega)}{p_{R}(\omega)-p_{r}(\omega)}\right) \\
& \quad \times\left(n\left(p_{R}(\omega), f_{\omega}(z)\right)+n\left(p_{R}(\omega), \frac{1}{f_{\omega}(z)}\right)\right) \rho_{n-1}(\omega) \\
& \quad+\frac{1}{r^{2 n-2}} \int_{\bar{B}_{n-1}(r)} \frac{2\left|\widetilde{q_{j}}-1\right| p_{r}(\omega) p_{R}(\omega)}{\left(p_{R}(\omega)-p_{r}(\omega)\right)\left(p_{R}(\omega)-|q| p_{r}(\omega)\right)} \\
& \quad \times\left(m\left(p_{R}(\omega), f_{\omega}(z)\right)+m\left(p_{R}(\omega), \frac{1}{f_{\omega}(z)}\right)\right) \rho_{n-1}(\omega) \\
& =S_{n}+S_{m},
\end{aligned}
$$

for $R>\max \left\{r,\left|\widetilde{q_{j}}\right| r\right\}$ and $0<\delta<1$. Now we will estimate terms $S_{m}$ and $S_{n}$, respectively. Note that

$$
\frac{p_{r}(\omega)}{p_{R}(\omega)} \leq \frac{r}{R}
$$

for $R>r$ and the fact that $x /(x-1)(x-t)$ is decreasing with $x$ for $x>\max \{1, t\}$. By using Lemma 4 , it follows that

$$
\begin{aligned}
S_{n} \leq & \left(\frac{R}{r}\right)^{2 n-2}\left(C\left(\widetilde{q_{j}}, \delta\right)+\frac{\left|\widetilde{q_{j}}-1\right| r}{R-\left|\widetilde{q_{j}}\right| r}+\frac{\left|\widetilde{q_{j}}-1\right| r}{R-r}\right) \\
& \times n_{f}(R, 0, \infty), \\
S_{m} \leq & \left(\frac{R}{r}\right)^{2 n-2} \frac{2\left|\widetilde{q_{j}}-1\right| r R}{(R-r)(R-|q| r)} m_{f}(R, 0, \infty),
\end{aligned}
$$

where

$$
\begin{aligned}
C\left(\widetilde{q_{j}}, \delta\right) & =\frac{\left|\widetilde{q_{j}}-1\right|^{\delta}\left(\left|\widetilde{q_{j}}\right|^{\delta}+1\right)}{\delta(1-\delta)\left|\widetilde{q_{j}}\right|^{\delta},} \\
n_{f}(R, 0, \infty) & =\left(n(R, f)+n\left(R, \frac{1}{f}\right)\right), \\
m_{f}(R, 0, \infty) & =\left(m(R, f)+m\left(R, \frac{1}{f}\right)\right) .
\end{aligned}
$$

It implies the assertion.

To deal with $m_{f}(R, 0, \infty)$, we need the following lemma.

Lemma 6 (see [14, Lemma 4]). If $T: \mathbb{R}^{+} \rightarrow \mathbb{R}^{+}$is a piecewise continuous increasing function such that

$$
\lim _{r \rightarrow \infty} \frac{\log T(r)}{\log r}=0
$$

then the set

$$
E:=\left\{r: T\left(C_{1} r\right) \geq C_{2} T(r)\right\}
$$

has logarithmic density 0 for all $C_{1}>1$ and $C_{2}>1$.

To show that $n_{f}(R, 0, \infty)$ is small, we need the following two lemmas.

Lemma 7 (see [12, Lemma 5.4]). Let $T: \mathbb{R}^{+} \rightarrow \mathbb{R}^{+}$be an increasing function, and let $U: \mathbb{R}^{+} \rightarrow \mathbb{R}^{+}$. If there exists a decreasing sequence $\left\{c_{n}\right\}_{n \in \mathbb{N}}$ such that $c_{n} \rightarrow 0$ as $n \rightarrow \infty$ and for all $n \in \mathbb{N}$, the set

$$
F_{n}:=\left\{r \geq 1: U(r)<c_{n} T(r)\right\}
$$

has logarithmic density 1, then

$$
U(r)=o(T(r))
$$

on a set of logarithmic density 1.

The following lemma is based on the proof of Lemma 5.3 in [12], which is the case of one dimension.

Lemma 8. If $f$ is a nonconstant meromorphic function in $\mathbb{C}^{n}$ of zero order, then the set

$$
E_{n}:=\left\{r \geq: n(r, f)<\frac{T(r, f)}{2^{n}}\right\}
$$

has logarithmic density 1 for all $n \in \mathbb{N}$.

If we take $R=k r$ in Lemma 5 , and by using Lemmas 6, 7, and 8 , then we have

$$
\int_{\partial B_{n}(r)} \log ^{+}\left|\frac{f\left(\widetilde{q_{j}} z\right)}{f(z)}\right| \sigma_{n}(z)=o(T(r, f))
$$


on a set of logarithmic density 1 , since $f$ is a meromorphic function in $\mathbb{C}^{n}$ of zero order. According to (23), it follows that

$$
\begin{aligned}
& \int_{\partial B_{n}(r)} \log ^{+}\left|\frac{f(q z)}{f(z)}\right| \sigma_{n}(z) \\
& \quad=\int_{\partial B_{n}(r)} \log ^{+} \prod_{k=1}^{n}\left|\frac{f\left(z \prod_{j=0}^{k} \widetilde{q_{j}}\right)}{f\left(z \prod_{j=0}^{k-1} \widetilde{q_{j}}\right)}\right| \sigma_{n}(z) \\
& \leq \sum_{k=1}^{n} \int_{\partial B_{n}(r)} \log ^{+}\left|\frac{f\left(z \prod_{j=0}^{k} \widetilde{q_{j}}\right)}{f\left(z \prod_{j=0}^{k-1} \widetilde{q_{j}}\right)}\right| \sigma_{n}(z)=o(T(r, f))
\end{aligned}
$$

on a set of logarithmic density 1 . Therefore, we have $q$ difference analogue of the lemma on logarithmic derivative of several variables, which is the proof of Theorem 1 .

\section{First and Second Main Theorems}

We will discuss the relation of $T(r, f(q z))$ and $T(r, f(z))$, which is the $q$-shift analogue of the first main theory of several variables.

Theorem 9. Let $f$ be a meromorphic function in $\mathbb{C}^{n}$ of zero order such that $f(0) \neq 0, \infty$, and let $q \in \mathbb{C}^{n} \backslash\{0\}$. Then

$$
T(r, f(q z))=T(r, f(z))+o(T(r, f))
$$

on a set of logarithmic density 1.

Proof. By using Lemma 6 and Theorem 1, we have

$$
\begin{aligned}
T(r, f(q z)) & \\
& =m(r, f(q z))+N(r, f(q z)) \\
& =m(r, f(z))+N(|q| r, f(z))+m\left(r, \frac{f(q z)}{f(z)}\right) \\
& \leq T(r, f)+o(T(r, f)),
\end{aligned}
$$

on a set of logarithmic density 1 . We have the assertion.

Let us discuss the $q$-shift analogue of the second main theory of several variables.

Theorem 10. Let $f$ be a meromorphic function in $\mathbb{C}^{n}$ of zero order such that $f(0) \neq 0, \infty$, and let $q \in \mathbb{C}^{n} \backslash\{0\}$. If $a_{i}(i=$ $1, \ldots, q) \in \mathbb{P}^{1}$ are distinct finite constants, then

$$
\begin{aligned}
& m(r, f)+\sum_{j=1}^{q} m\left(r, \frac{1}{f-a_{j}}\right) \\
& \leq 2 T(r, f)-N_{\text {pair }}(r, f)+S_{q}(r, f),
\end{aligned}
$$

where

$$
N_{\text {pair }}(r, f):=2 N(r, f)-N\left(r, \Delta_{q} f\right)+N\left(r, \frac{1}{\Delta_{q} f}\right) .
$$

Proof. By using the first main theorem, we obtain

$$
\begin{aligned}
\sum_{j=1}^{q} m\left(r, \frac{1}{f-a_{j}}\right) & =\sum_{j=1}^{q} T\left(r, \frac{1}{f-a_{j}}\right)-\sum_{j=1}^{q} N\left(r, \frac{1}{f-a_{j}}\right) \\
& =q T(r, f)-N\left(r, \frac{1}{P(f)}\right)+S_{q}(r, f),
\end{aligned}
$$

where

$$
P(r, f)=\prod_{j=1}^{q}\left(f-a_{j}\right) .
$$

Since there exist $\alpha_{j} \in \mathbb{P}^{1}$ such that

$$
\frac{1}{P(f)}=\sum_{j=1}^{q} \frac{\alpha_{j}}{f-a_{j}}
$$

then

$$
\begin{aligned}
m\left(r, \frac{1}{P(f)}\right) & \leq m\left(r, \frac{\Delta_{q} f}{P(f)}\right)+m\left(r, \frac{1}{\Delta_{q} f}\right) \\
& \leq m\left(r, \frac{1}{\Delta_{q} f}\right)+\sum_{j=1}^{q} m\left(r, \frac{\Delta_{q} f}{f-a_{j}}\right) \\
& =m\left(r, \frac{1}{\Delta_{q} f}\right)+S_{q}(r, f) .
\end{aligned}
$$

By applying (29), (32), and the fact $T(r, P(f))=q T(r, f)+$ $O(1)$, we have

$$
\begin{aligned}
\sum_{j=1}^{q} m\left(r, \frac{1}{f-a_{j}}\right) \\
\quad=T(r, P(f))-N\left(r, \frac{1}{P(f)}\right)+S_{q}(r, f) \\
\quad=m\left(r, \frac{1}{P(f)}\right)+S_{q}(r, f) \\
\quad \leq m\left(r, \frac{1}{\Delta_{q} f}\right)+S_{q}(r, f) \\
\quad=T\left(r, \Delta_{q} f\right)-N\left(r, \frac{1}{\Delta_{q} f}\right)+S_{q}(r, f) .
\end{aligned}
$$

Therefore, we have

$$
\begin{aligned}
m(r, f) & +\sum_{j=1}^{q} m\left(r, \frac{1}{f-a_{j}}\right) \\
\leq & T(r, f)+N\left(r, \Delta_{q} f\right)+m\left(r, \Delta_{q} f\right) \\
& -N\left(r, \frac{1}{\Delta_{q} f}\right)-N(r, f)+S_{q}(r, f) .
\end{aligned}
$$


Since

$$
\begin{aligned}
m\left(r, \Delta_{q} f\right) & \leq m(r, f)+m\left(r, \frac{\Delta_{q} f}{f}\right) \\
& =m(r, f)+S_{q}(r, f),
\end{aligned}
$$

we have the assertion.

\section{Application}

Let $f$ and $g$ be two meromorphic functions in $\mathbb{C}^{n}$, and let $a \in \mathbb{C}^{n}$, if $f-a$ and $g-a$ have the same zeros (counting multiplicities), then we say $f$ and $g$ share $a \mathrm{CM}$ in $\mathbb{C}^{n}$.

Theorem 11. Let $f: \mathbb{C}^{n} \rightarrow \mathbb{P}^{1}$ be a nonconstant meromorphic function of zero order and let $q \in \mathbb{C}^{n} \backslash\{0\}$. If $f(z)$ and $f(q z)$ share three distinct values $C M$, then $f(z)=f(q z)$.

Proof. Let us suppose that $f(z)$ and $f(q z)$ share $\{0,1, \infty\}$ CM, if not, let us make a linearly transformation. Suppose that $\Delta_{q} f \neq \equiv$, Theorem 10 yields

$$
\begin{aligned}
m(r, f) & +m\left(r, \frac{1}{f}\right)+m\left(r, \frac{1}{f-1}\right) \\
\leq & 2 T(r, f)+N\left(r, \Delta_{q} f\right)-2 N(r, f)-N\left(r, \frac{1}{\Delta_{q} f}\right) \\
& +S_{q}(r, f) .
\end{aligned}
$$

Since $f(z)$ and $f(q z)$ share $\{0,1, \infty\} \mathrm{CM}$, then

$$
N(r, f)+N\left(r, \frac{1}{f}\right)+N\left(r, \frac{1}{f-1}\right) \leq N\left(r, \frac{1}{\Delta_{q} f}\right) .
$$

In addition, Lemma 6 implies that $N\left(r, \Delta_{q} f\right) \leq 2 N(r, f)+$ $S_{q}(r, f)$, by combining (36) and (37), it follows that

$$
T(r, f)=S_{q}(r, f)
$$

which is impossible.

The $q$-difference polynomials of $f(z)$ in $\mathbb{C}^{n}$ are said by the functions which are polynomials in $f\left(q_{j} z\right)$, where $q_{j} \in \mathbb{C}^{n}$, with coefficients $a_{\lambda}(z)$ such that $T\left(r, a_{\lambda}(z)\right)=o(T(r, f))$ on a set of logarithmic density 1 .

The following theorem is $q$-shift analogue of Clunie lemma [15] of several variables.

Theorem 12. Let $f: \mathbb{C}^{n} \rightarrow \mathbb{P}^{1}$ be a nonconstant zero order meromorphic solution in $\mathbb{C}^{n}$ of

$$
f(z)^{n} P(z, f)=Q(z, f)
$$

where $P(z, f)$ and $Q(z, f)$ are q-difference polynomials in $f(z)$. If the degree of $Q(z, f)$ as a polynomial in $f(z)$ and its $q$-shift is at most $n$, then

$$
m(r, P(z, f))=S_{q}(r, f) .
$$

Proof. In calculating the proximity function of $P(z, f)$, we split the region of integration into two parts. By defining

$$
E_{1}:=\left\{z \in \partial B_{n}(r)|| f(z) \mid<1\right\}, \quad E_{2}:=\partial B_{n}(r) \backslash E_{1},
$$

we have

$$
\begin{aligned}
m(r, P(z, f))= & \int_{E_{1}} \log ^{+}|P(z, f)| \sigma_{n}(z) \\
& +\int_{E_{2}} \log ^{+}|P(z, f)| \sigma_{n}(z) .
\end{aligned}
$$

First, we consider $E_{1}$; we can write $P(z, f)$ as

$$
P(z, f)=\sum c_{i}(z) f(z)^{i_{0}} f(q z)^{i_{1}} \cdots f\left(q_{\nu} z\right)^{l_{\nu}} .
$$

Thus, we have

$$
|P(z, f)| \leq \sum\left|c_{i}(z)\right|\left|\frac{f(q z)^{i_{1}}}{f(z)}\right|^{l_{1}} \ldots\left|\frac{f\left(q_{\nu} z\right)^{l_{\nu}}}{f(z)}\right| .
$$

Therefore, we obtain

$$
\begin{aligned}
& \int_{E_{1}} \log ^{+}|P(z, f)| \sigma_{n}(z) \\
& \quad \leq \sum m\left(r, c_{i}\right)+O\left(\sum_{i=1}^{q} m\left(r, \frac{f(q z)}{f(z)}\right)\right),
\end{aligned}
$$

together with Theorem 1 implies that

$$
\int_{E_{1}} \log ^{+}|P(z, f)| \sigma_{n}(z)=S_{q}(r, f) .
$$

Now let us consider $E_{2}$; we note that

$$
Q(z, f)=\sum b_{\gamma}(z) f\left(q_{0} z\right)^{\gamma_{0}} \cdots f\left(q_{\mu} z\right)^{\gamma_{\mu}} .
$$

Hence, we have

$$
\begin{aligned}
|P(z, f)| & =\left|\frac{1}{f(z)^{n}} \sum b_{\gamma}(z) f\left(q_{0} z\right)^{\gamma_{0}} \cdots f\left(q_{\mu} z\right)^{\gamma_{\mu}}\right| \\
& \leq \sum\left|b_{\gamma}(z)\right|\left|\frac{f\left(q_{1} z\right)}{f(z)}\right|^{\gamma_{1}} \cdots\left|\frac{f\left(q_{\mu} z\right)}{f(z)}\right|^{\gamma_{\mu}},
\end{aligned}
$$

by Theorem 1 again, it follows that

$$
\int_{E_{2}} \log ^{+}|P(z, f)| \sigma_{n}(z)=S_{q}(r, f) .
$$

The assertion follows by combining (42)-(49).

Let $\alpha$ and $f$ be meromorphic functions of zero order in $\mathbb{C}^{n}$ such that $T(r, \alpha)=o(T(r, f))$ on a set of logarithmic density 1. Then $\alpha$ is called a small function with respect to $f$ of zero order in $\mathbb{C}^{n}$.

The following theorem is $q$-shift analogue of Mohon'ho lemma [16] of several variables. 
Theorem 13. Let $f(z)$ be a nonconstant zero-order meromorphic solution in $\mathbb{C}^{n}$ of

$$
P(z, f)=0,
$$

where $P(z, f)$ is a q-difference polynomial in $f(z)$. If $P(z, \alpha)$ \# 0 for a small function $\alpha$ in $\mathbb{C}^{n}$ of $f(z)$, then

$$
m\left(r, \frac{1}{f-\alpha}\right)=S_{q}(r, f) .
$$

Proof. By substituting $f=g+\alpha$ into (50), we obtain

$$
Q(z, g)+D(z)=0,
$$

where

$$
Q(z, g)=\sum_{\gamma=\left(j_{0}, \ldots, j_{\nu}\right) \in J} b_{\nu}(z) g(z)^{j_{0}} \cdots g\left(q_{v} z\right)^{j_{\nu}}
$$

is a $q$-difference polynomial in $g$ such that all of its terms are at least degree 1 , and $T(r, D)=S_{q}(r, g), T\left(r, b_{v}(z)\right)=S_{q}(r, g)$ for each $\nu \in J$. Also $D \neq \equiv 0$, since $\alpha$ does not satisfy (50). By using (52), we have

$$
\begin{aligned}
m\left(r, \frac{1}{g}\right) & \leq m\left(r, \frac{D}{g}\right)+m\left(r, \frac{1}{D}\right) \\
& =m\left(r, \frac{Q(z, g)}{g}\right)+m\left(r, \frac{1}{D}\right) .
\end{aligned}
$$

Note that since the integral $m(r, 1 / g)$ vanishes on the part of $\partial B_{n}(r)$ where $|g|>1$, it is sufficient to consider only the case $|g| \leq 1$. Then

$$
\begin{aligned}
\left|\frac{Q(z, g)}{g}\right| & =\frac{1}{|g|}\left|\sum_{\gamma \in J} b_{\nu}(z) g(z)^{j_{0}} \cdots g\left(q_{\nu} z\right)^{j_{\nu}}\right| \\
& \leq \sum_{\gamma \in J}\left|b_{\nu}(z)\right|\left|\frac{g\left(q_{1} z\right)}{g(z)}\right|^{j_{1}} \cdots\left|\frac{g\left(q_{\nu} z\right)}{g(z)}\right|^{j_{\nu}},
\end{aligned}
$$

by Theorem 1, (54), and (55), it shows

$$
m\left(r, \frac{1}{g}\right)=S_{q}(r, g) .
$$

Since $g=f-\alpha$, the assertion follows.

\section{Conflict of Interests}

The author declares that there is no conflict of interests regarding the publication of this paper.

\section{References}

[1] W. K. Hayman, Meromorphic Functions, Oxford Mathematical Monographs, Clarendon Press, Oxford, UK, 1964.

[2] C.-C. Yang and H.-X. Yi, Uniqueness Theory of Meromorphic Functions, vol. 557 of Mathematics and Its Applications, Kluwer Academic Publishers, Dordrecht, The Netherlands, 2003.
[3] I. Laine, Nevanlinna Theory and Complex Differential Equations, vol. 15 of de Gruyter Studies in Mathematics, Walter de Gruyter, Berlin, Germany, 1993.

[4] A. Vitter, "The lemma of the logarithmic derivative in several complex variables," Duke Mathematical Journal, vol. 44, no. 1, pp. 89-104, 1977.

[5] A. Biancofiore and W. Stoll, "Another proof of the lemma of the logarithmic derivative in several complex variables," in Recent Developments in Several Complex Variables (Proc. Conf., Princeton Univ., Princeton, N. J., 1979), vol. 100 of Annals of Mathematics Studies, pp. 29-45, Princeton University Press, Princeton, NJ, USA, 1981.

[6] Z. Ye, "A sharp form of Nevanlinna's second main theorem of several complex variables," Mathematische Zeitschrift, vol. 222, no. 1, pp. 81-95, 1996.

[7] B. Q. Li, "A logarithmic derivative lemma in several complex variables and its applications," Transactions of the American Mathematical Society, vol. 363, no. 12, pp. 6257-6267, 2011.

[8] R. G. Halburd and R. J. Korhonen, "Nevanlinna theory for the difference operator," Annales Academiae Scientiarum Fennicae: Mathematica, vol. 31, no. 2, pp. 463-478, 2006.

[9] R. G. Halburd and R. J. Korhonen, "Difference analogue of the lemma on the logarithmic derivative with applications to difference equations," Journal of Mathematical Analysis and Applications, vol. 314, no. 2, pp. 477-487, 2006.

[10] R. G. Halburd, R. Korhonen, and K. Tohge, "Holomorphic curves with shift-invariant hyperplane preimages," Transactions of the American Mathematical Society, vol. 366, pp. 4267-4298, 2014.

[11] R. Korhonen, "A difference Picard theorem for meromorphic functions of several variables," Computational Methods and Function Theory, vol. 12, no. 1, pp. 343-361, 2012.

[12] D. C. Barnett, R. G. Halburd, W. Morgan, and R. J. Korhonen, "Nevanlinna theory for the $q$-difference operator and meromorphic solutions of $q$-difference equations," Proceedings of the Royal Society of Edinburgh A: Mathematics, vol. 137, no. 3, pp. 457-474, 2007.

[13] P.-C. Hu, P. Li, and C.-C. Yang, Unicity of Meromorphic Mappings, vol. 1 of Advances in Complex Analysis and Its Applications, Kluwer Academic Publishers, Dordrecht, The Netherlands, 2003.

[14] W. K. Hayman, "On the characteristic of functions meromorphic in the plane and of their integrals," Proceedings of the London Mathematical Society A, vol. 14, pp. 93-128, 1965.

[15] J. Clunie, "On integral and meromorphic functions," Journal of the London Mathematical Society, vol. 37, pp. 17-27, 1962.

[16] A. A. Mohon'ko and V. D. Mohon'ko, "Estimates of the Nevanlinna characteristics of certain classes of meromorphic functions, and their applications to differential equations," Sibirskiŭ Matematičeskiǔ Zurnal, vol. 15, pp. 1305-1322, 1431, 1974. 


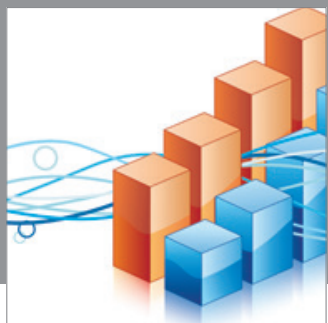

Advances in

Operations Research

mansans

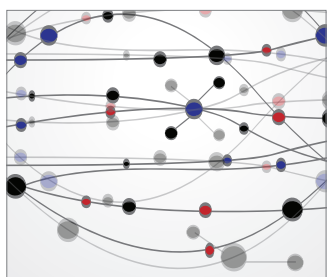

The Scientific World Journal
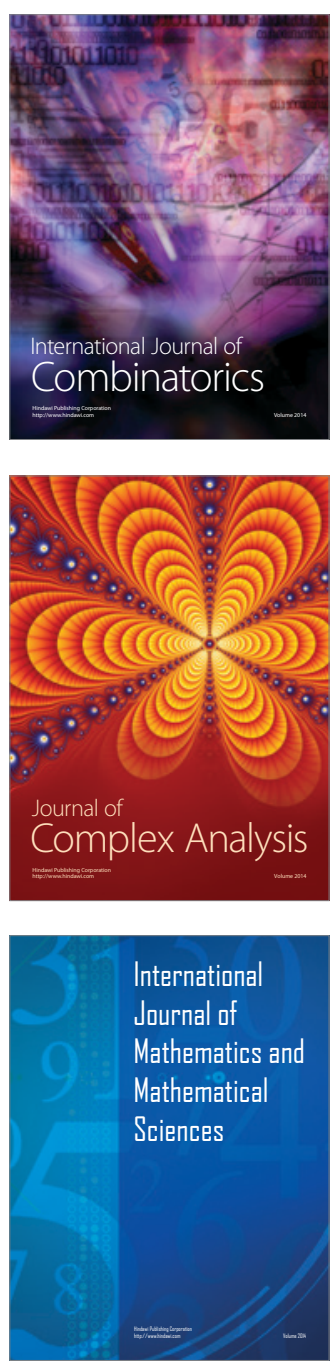
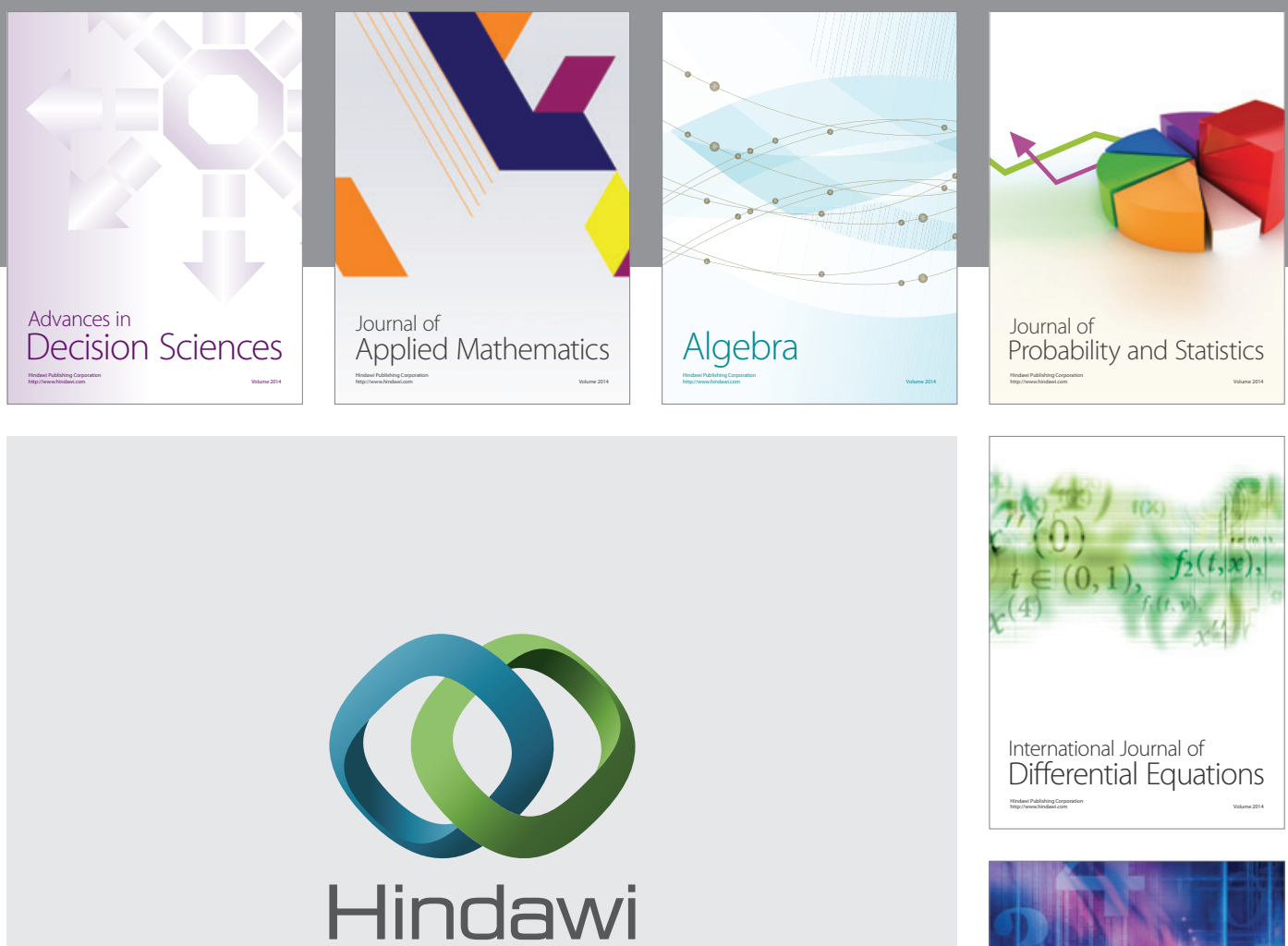

Submit your manuscripts at http://www.hindawi.com
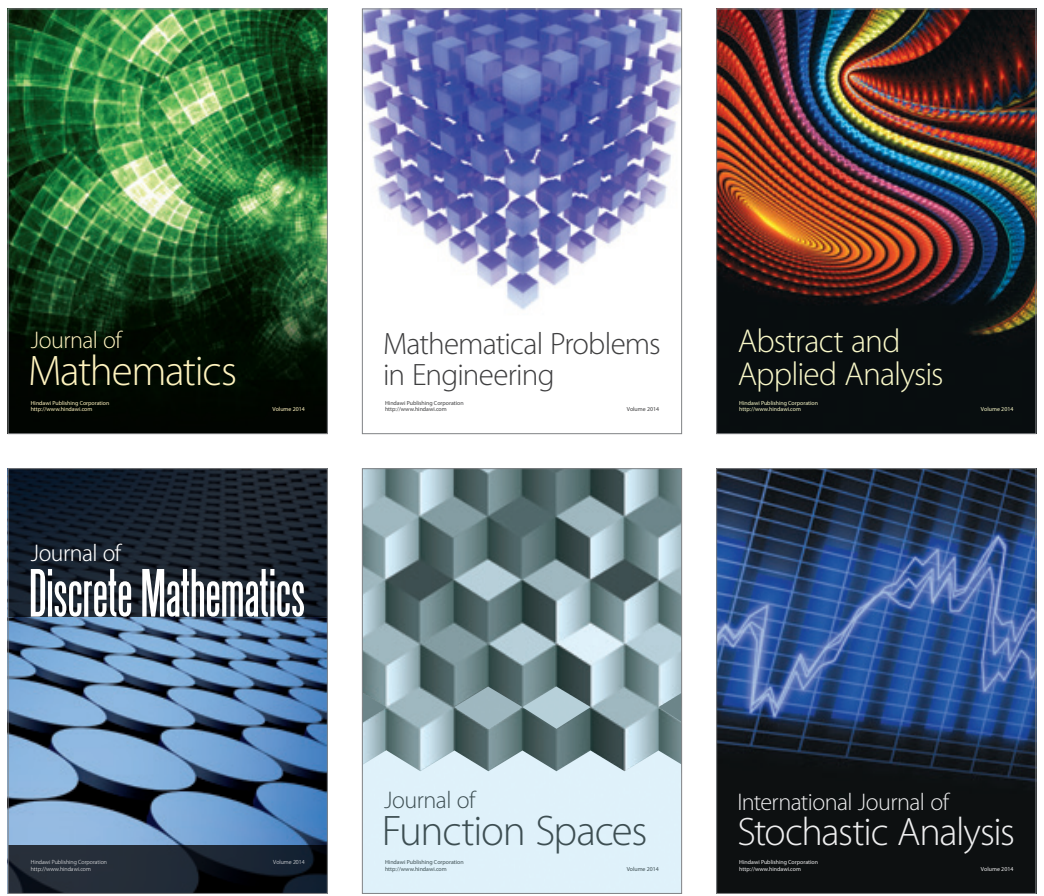

Journal of

Function Spaces

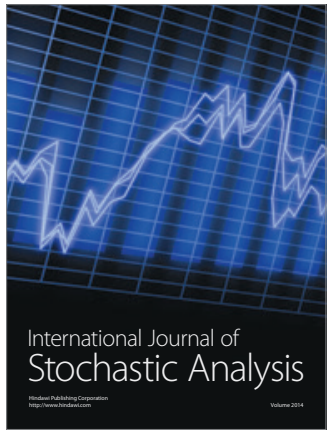

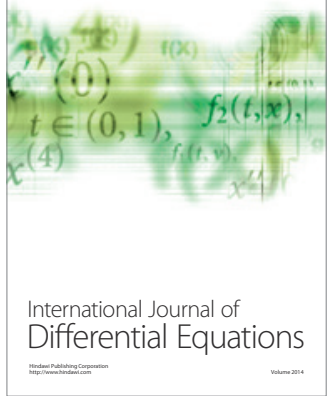
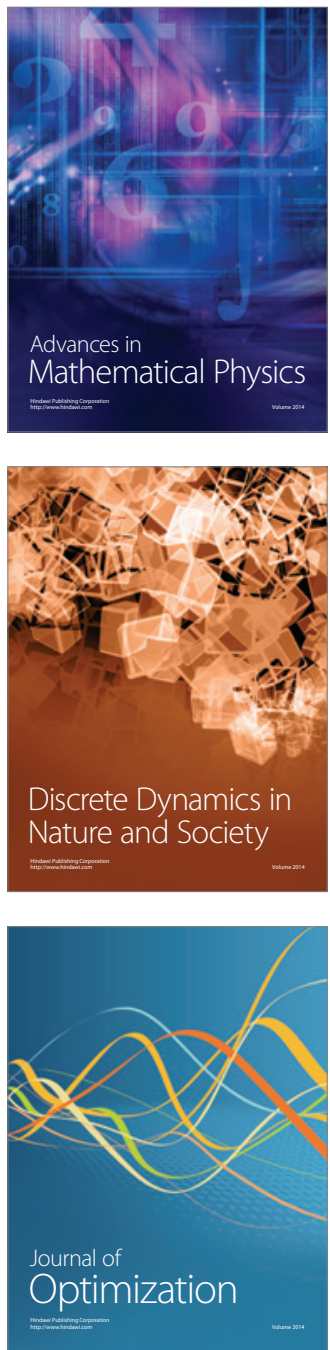